\title{
Building and Applying a Human Cognition Model for Visual Analytics
}

\author{
Tera Marie Green 1 , William Ribarsky', \& Brian Fisher ${ }^{2}$ \\ ${ }^{1}$ Charlotte Visualization Center, University of North Carolina at Charlotte \\ ${ }^{2}$ School of Interactive Arts and Technology, Simon Fraser University
}

\begin{abstract}
It is well known that visual analytics addresses the difficulty of evaluating and processing large quantities of information. Less often discussed are the increasingly complex analytic and reasoning processes that must be applied in order to accomplish that goal. Success of the visual analytics approach will require us to develop new visualization models that predict how computational processes might facilitate human insight and guide the flow of human reasoning. In this paper, we seek to advance visualization methods by proposing a framework for human "higher cognition" that extends more familiar perceptual models. Based on this approach, we suggest guidelines for the development of visual interfaces that better integrate complementary capabilities of humans and computers. While many of these recommendations are novel, some can be found in existing visual analytics applications. In the latter case, much of the value of our contribution lies in the deeper rationale that the model provides for those principles. We then assess these visual analytics guidelines through the evaluation of several visualization examples. Lastly, we discuss steps that can be taken towards a predictive human cognition model.
\end{abstract}

KEYWORDS: visual analytics, cognition and perception theory, embodied cognition, visualization taxonomies and models 


\section{INTRODUCTION}

In a previous paper [1], we discussed the value of considering performance characteristics of human reasoning, problem-solving, and decision-making, in addition to the more familiar perceptual processes, to develop information and knowledge visualizations. The driving force behind this program of research is the need to develop applications capable of attacking today's complex and critical problems with the required depth of reasoning and analysis that will support their solution. This paper builds on the work reported in our VAST 2008 paper [41] and expands our argument that the creation of comprehensive models of human-computer cognitive processing should be a core component of the visual analytics effort, and is an essential prerequisite for success of visual analytics as a field. In most visualization development "higher cognition" processes have been considered as a "black box" that receives information from the visualization through a perceptual transduction filter, and that generates responsive behavior through unspecified internal mechanisms. (We will discuss this metaphorical view of humanvisualization interaction during our consideration of the van Wijk operational model of visualization in Section 4.) However, as both interactive visualizations and cognitive tasks become semantically as well as perceptually complex, it becomes apparent that we must peek into the black box in an attempt to model and predict the behavior of the human-computer collaboration as a cognitive system. Psychology and related behavioral sciences have examined reasoning and other thought processes for decades, through classical scientific processes of reduction, laboratory testing, and scientific induction. One reason that much of this research has, as yet, been unused in the construction of interactive visualizations is the lack of a broad theory of human reasoning with sufficient scope and predictive validity to impact the design and evaluation of those applications. It is, as Newell once wrote, as if "science advances by playing twenty questions with nature" [2]. As Newell suggests, the reductionist approach to the study of higher cognition creates multiple competing theories of small, often binary, aspects of reasoning, and these have dominated the research. His solution to this problem was to 
propose that cognitive scientists generate models of large scale "cognitive architecture" such as his own SOAR [Unified theories of cognition ref], and Anderson's ACT-R [An Integrated Theory of the Mind]. These models have had some success at modeling human abstract problem solving but have struggled to accommodate cognitive activities that depend upon perceptual or motor processes to a high degree, such as fluent human interaction with a visualization system in the course of analyzing a situation and solving a problem.

But while complex, higher cognition is still predictable to some degree. The number of available heuristics is finite, and is therefore theoretically knowable. And while extant research may disagree on the finer points, there is general agreement that humans are parsimonious problem solvers who most frequently choose to use the simplest heuristic that is adequate to accomplish a given task. For our purposes it may be sufficient to find aspects of human cognition that are sufficiently predictive that they can constrain the range of possible designs, and propose metrics for testing the success of those methods. This is a simpler task than the comprehensive model of human information processing that cognitive science seeks, although progress towards our goals may well have an impact on those more comprehensive models of human cognition.

This paper endeavors to lay the framework of a human cognition model, whose guidelines would guide the development of visual interfaces more able to attack the complex problems now being faced by analysts and researchers. Additionally, this paper shows how this model contributes new, cognition-based principles of visualization design. Some of these principles are already being used in the better visual analytics designs, but without the deeper rationale that the model provides. We will discuss and evaluate these visual analytics methods. Other principles from the model have not been applied or not fully applied, and we will discuss how their implementation and use will be of benefit. 


\section{COMPLEMENTARY STRENGTHS}

Our focus is on mixed-initiative visualization, in which both the computer and the human initiate processes and with which both collaborate in the exploration and creation of knowledge. Both human and computer bring strengths to the overall cognitive system. Several of the obvious strengths are complementary, which further strengthens the potential of the collaboration.

\section{Human strengths}

Some of the earliest reasoning skills humans develop are those of adaptation and accommodation [3]. Adaptation is the ability to incorporate newly perceived information into extant knowledge schema, and it relies heavily on our ability to categorize sensory stimuli at a rate that is nearly instantaneous. Even when what is perceived is so novel it will not fit existing knowledge schema, accommodation allows a human to temporarily place a marker in a closely similar schema or create a new one [4]. This "fast and frugal" reasoning ability [5] enables humans to more effectively deal with rapidly-changing situations. Biederman's 1987 Recognition By Components (RBC) model provides a mechanism by which basic-level categorization of visual objects takes place rapidly and accurately regardless of viewpoint or changes in nonessential characteristics of those objects. [6]. This process is effortless (i.e. it does not demand attentional resources) for a human, and it allows the reasoning process to advance despite incomplete information. It is far superior to current computer object recognition. In addition to our ability to categorize individual objects, human perceptual abilities are also well adapted to complex and rapidly changing scenes, defined here as complex sets of objects and events distributed in space that interact with each other in often novel ways. 
Scene perception takes place through an integrated set of mechanisms that optimize performance under the constraints of limitations in central resources such as focal attention. Scene processing begins with a fast low-level "gist" mechanism that recognizes important scene characteristics and relationships [7]. This preattentive visual process guides the allocation of multiple attentional tokens known as FINSTs, see [8, 9] that support the rapid and automatic calculation of a set of operations [10] known as visual routines that relay information on their properties and relations to each other. All of this occurs prior to, and in support of, endogenous focal attention (i.e. attention to the task at hand). This resulting cascade of processes frees cognition for consideration of higher order characteristics of the information contained in the display rather than the display itself -- object properties and spatial relations with other objects and causal relations to events taking place. Thus, cognitive operations can proceed using more parsimonious representations that are well- suited for the task at hand. This can be thought of as a two-step process by which unconscious inference, the "logic of perception", [11] works hand-in-hand with cognitive processes to support reasoning. In this view, expertise is not only a characteristic of higher-order cognitive logic, but also of perceptual logic, which can be trained to better support cognitive operations through "perceptual expertise" [12].

Partially due to a lifetime of experience in adaptation and accommodation, humans are much more flexible reasoners than AI models, and in particular much better enabled to understand novel situations and novel approaches to known problems. Humans rely on a compendium of reasoning and problem-solving heuristics, which can be used singly or concomitantly to accomplish the task at hand. The simplest of these, elimination heuristics such as satisficing [13], eliminate available choices that do not possess an identified important attribute. Elimination heuristics require little cognitive effort, and so are often what a human will use first in an attempt to narrow down available choices. 
Of course, if the problem becomes semantically complex, more effort is required. Our model assumes a mental model to inferred rules mechanization [14], wherein the human uses all available information to create a mental model of the concept being considered. From this model, the human infers generalizeable rules - sometimes in a matter of seconds - that are used in later instantiations of a similar concept or problem. Because these models are based entirely on available (including previously held) information, it is imperative that all pertinent information is available to avoid the creation of incomplete mental models, which are, in turn, likely to be the basis of invalid rules.

\section{Computer strengths}

A computer is capable of two distinct processes that complement human reasoning strengths well: superior working memory and information processing without cognitive biases. Humans depend on their working memory as they reason, but are, at best, able to remember $7 \pm 2$ chunks of information [15]. The computer, on the other hand, has a "working memory" limited only by hardware. The computer's ability to keep all pertinent information visually available to the human aids in complete mental modeling, among other things. The other computer strength is the lack of inherent biases. This bias-free environment is, to be sure, influenced by what the interface is designed to see as relationally relevant. But unlike humans, computers do not situationally filter out pertinent information in accordance with a perceived belief or due to the way a problem is presented $[16,17]$. By presenting all relevant information, the computer can aid not only in mental modeling, but also in the analysis of competing hypotheses.

\section{USE OF A HUMAN COGNITION MODEL}

This section will discuss the ways in which knowledge of higher cognition focuses a mixedinitiative human cognition model $(\mathrm{HCM})$, as well as provides several guidelines which can be derived from the model's use. (See Figure 1.) On each of the HCM's submodels, please see [1] for more discussion. 


\section{Information Discovery \& Knowledge Building}

The central process of the HCM is discovery, during which the computer presents information in an ontological-like structure within a relevant, possibly human-defined, context. Presenting information with a relevant context is one method of mitigating human cognitive overload in the midst of an overwhelming number of semantic data points. The human directly interacts with the visualized information, focusing the attention of discovery. We will explore this idea further in

\section{Section 5.4.}

An intuitive multi-model visualization also encourages knowledge building through new knowledge creation. Throughout the process of discovery, the human may uncover a relationship between two currently unrelated concepts or ideas. By creating a new relationship between the two concepts and perhaps annotating the relationship, the human collaborator can extend the knowledge base of the visualization, not only for what is to be accomplished in that particular session, but for every session by every human who uses the visualization thereafter.

The computer can augment the discovery of relevant information through computer-aided discovery. Through observation of what interests the human collaborator, the computer can suggest information that is semantically related, but up to this point, has not been considered. This also would include relational data which has been added by other human collaborators, which allows one person to learn from another. The human is free to explore or to reject the suggestion. But by making the effort to ensure that nothing important is overlooked, the computer works to counteract human cognitive biases which can interfere with complete mental modeling. 


\section{Guidelines for Discovery and Knowledge Building}

We will now briefly discuss several guidelines based upon the HCM discovery and knowledge submodels. These can be used to motivate visual analytics interface design.

\section{Multiple views}

When the information being explored is semantically rich, and could be visualized through a variety of categorization levels, it is often left to the discretion of the visualization developer as to which level merits the primary view. It is important to categorize information to aid the human in directing attention, but we would argue that a visualization that utilizes multiple organizational views of the same information can be a powerful aid. As the human interacts with information in any view, the relational changes are visualized in all views. While the concept of multiple views is not a new one [18], what we would highlight is how multiple views are informed by human cognition. First, as humans perceive information in a variety of ways including through the filter of their own assumptions, patterns are more likely to be discovered if represented multiple ways, each tuned to particular, important aspects of the data.

Secondly, as we have discussed previously, humans prefer to narrow down the field of choices by eliminating those that do not posses desired attributes. This is usually done before utilizing more complicated heuristics. Multiple views make the process easier; multiple layers of relational attributes are readily knowable without additional search.

Thirdly, multiple views enable more intuitive manipulation. Humans themselves do not interact with information in one dimension; humans are capable of multi-layered processing: perceptual, emotional, and higher-cognitive. Indeed, of all the guidelines we will discuss, use of multiple views is the one most likely to lead to spontaneous insight.

Lastly, the multiple views can be each set for different cognitive viewpoints or cognitive tasks as part of the overall problem-solving approach. This can be done in a general way, as is shown in the WireVis example below. 


\section{Direct interaction}

By definition, a well-designed information visualization allows the user to directly interact with information. But we would take direct interaction one step further. In computer-aided reasoning and discovery, for example, the guideline of direct interaction would propose that whatever tactic the computer uses to suggest relational information to the human be done without interfering with a human's train of thought or flow of reasoning. Additionally, direct interaction supports the goals of other HCM guidelines by facilitating rich, fast, and effective interaction. The human thinks in terms of the analysis task, which is closely aligned with the interaction, and then looks at the visualized results. As a result, the user is more able to stay in the cognitive zone (as we will discuss shortly), even with multiple windows. With this in mind, visualization design should avoid, as much as possible, menus or other actions that take the user outside of the frame of the task. Interactions should be through direct manipulation and translucent wherever possible, avoiding the traditional pull-down menus, which require the human to sort through and think about menu items.

\section{Central Role of Interaction}

Human-computer interaction is not a series of disjointed behaviors. And while the visual process is an important part of visualization, it does not stand alone. Interaction has a rhythm, a cycle of give and take. Interaction is the process by which the computer and the human give, take, and create knowledge. We will see an example of this when we will consider the van Wijk operational model in the next section. 
Insulation of Reasoning Flow

One goal of intuitive visualization should be the facilitation of the flow of human reasoning. Once the human has focused cognitive resources in an area of interest, the visualization should not hamper the rhythm of reasoning until the human chooses to refocus resources elsewhere. This insulation can be achieved partially through direct interaction within context and intuitive computer-aided information discovery, as is discussed elsewhere in this section. Insulation is also aided, where possible, by an understanding of the temporal constraints of human perception and patterns of cognitive activity, adapting the timing of interface events and/or reducing the time required to retrieve necessary information from interface interaction [19].

Spivey [37] argues that the pace of cognitive operations is determined by the need to reduce the impact of the time required to pull information from the world on the flow of cognitive processing. This is due to the high cost of storing and retrieving items in working memory. Given the poor buffering capabilities of human information processing, it is more effective to simply reduce the speed of cognitive processes to match those of information uptake. For example, humans can think through visual material only as quickly as the eyes can move. In fact, the time that is required to read text is much faster if each word in the sentence is displayed in series at the same position in space (the Rapid Serial Visual Presentation technique). In normal reading, the pace of information processing slows to allow the eyes to move to the next word before the word just read is fully processed. Thus, the experience of reading shifts from a read-rememberread stop-and-go rhythm to a continuous (but slower) flow of reading. This insulates cognition from the stop-and-go of eye movements, and the experience seems continuous. 
For visualization, especially interactive visualization, the temporal constraints of the perception and control of the image differ. To optimize the use of cognitive resources, designers need to understand what temporal pattern of mental activity should take place for optimal cognitive performance, and adapt the timing of events so as to maintain that pattern. This will require additional research on rhythms of human information processing that are sufficiently predictive to generate guidelines that can be applied in the design process, perhaps by an attentive system that monitors user actions (such as eye movements) to infer patterns of cognitive processes.

In the terminology of the HCM, being "in the zone" allows the human collaborator to reason without encountering unnecessary attentional or cognitive impediments. In cases where task complexity exceeds the user's ability to process information, or a cognitive impasse is reached for some other reason, the computer can provide a scaffolding of support by presenting the information within relevant context, suggesting what may have been overlooked, and keeping relevant information present.

Intimate interaction

It is important that the interaction is so translucent to the human collaborator that the give and take which occurs in a successful collaboration is seamless. Entering the interaction should seem natural and obvious. The use of on-screen tools should not require additional cognitive focus - i.e. be usable without the human having to "think about it." Intimate interaction deters attentional interference during the cognitive flow, and enables the reasoning process to move forward unabated. When interaction is intimate, what the human should see and cognitively manipulate is not the tool being used or the method of interaction, but only the interaction itself. Intimate interaction is an important asset to flow insulation, and is supportive of the central role of interaction. 


\section{Search by Example \& Search by Pattern}

Searching for information has traditionally been done by entering a search term in a text box. But text boxes require humans to specify what to look for (such as in Boolean-type queries), as well as to stop where they are in the reasoning process to formulate a query in concrete terms. Finding an appropriate Boolean query will almost always break the flow of cognitive processing. We would argue that a better general approach would be to allow the human, where possible and appropriate, to indicate the search terms by pointing and clicking on an example or drawing a bounding box around a pattern of examples or other relational information. This allows the human to indicate the search visually, without the burden of linguistic encoding. This does not require an interruption in thought, feels more intuitive, and allows reasoning to move forward.

It is true that Boolean searches can be necessary under certain conditions. For example, working within the definition of an information scent model [20], a point-and-click search-byexample is likely a better approach. But if the user exhausts an old scent, coding a Boolean query becomes necessary in the hunt for a new one. Key here is to make the decision about which type of search to employ based on the prediction of its impact on cognition. One of the interfaces we consider in the Examples section, WireVis, has successfully used point-and-click search by example with one or more variables. This, of course, requires underpinning analytical tools tightly integrated with the visual interface. This might not always be feasible, but it is a main goal of visual analytics. 


\section{Creation and Analysis of Hypotheses}

One extension of the knowledge building process that holds great potential for multi-modal visualization is in the creation and evaluation of hypotheses. Hypothesis generation and evaluation is highly impacted by human cognitive bias, as humans are wont to seek out confirmatory evidence rather than to seek disconfirmation.

As Heuer described it [21], hypothesis analysis starts with a list of hypotheses and a body of evidence that proves or disproves each one. As the human creates of list of hypotheses, the computer can aid in finding relevant evidence. From there, the computer, with its superior working memory, creates a weighted matrix or similar relational structure that the human can evaluate with her superior reasoning ability. Using the edited relational structure, the human draws conclusions about which hypotheses are correct, and if desired, sets up a data watch in the visualization that will notify the user of data changes. Hypotheses generation is initiated by the human, but the computer plays a significant role in shortening the process and neutralizing biases, contributing to more solid conclusion through use of its strengths.

\section{CONSIDERING THE VAN WIJK MODEL}

We will now discuss what the implications of our model would look like if integrated into the van Wijk operational model of the visualization process [22]. We do this primarily as another way to envision how a human cognition model interrelates with other aspects of visualization theory, or as another way to broadly sketch out the basic assumptions of the HCM within the context of an extant model.

Van Wijk models the "user" as $\mathrm{P}$ (perception and cognition), $\mathrm{K}$ (knowledge), and $\mathrm{E}$ (interactive exploration), as is demonstrated in Figure 2. The user perceives the image (I) and interacts within the visualization using a variety of available manipulation techniques (the specifications (S)). 
The model depicts Perception as feeding Knowledge, which, in turn, drives interactive Exploration. This is appropriate for aspects of human perception, such as what Gray calls “microstrategies"[36] -- simple perception/action patterns that take place largely without conscious direction but under executive control of cognitive processes. Some perceptions are not affected by the specifics of what the user knows, plans, and expects; these are aspects of performance we can depend on to remain more or less constant, or "cognitively impenetrable" $[8,9]$.

There is still some discussion about which perceptual tasks could be considered truly "preattentive" and which evidence cognitive manipulation (see for example [37]), and it is not our intent to explore this debate in any depth here. Our intent is to demonstrate the cooperative give-and-take of cognition throughout interactive visualization exploration. Thus, for the purposes of this control diagram, we will define Perception to include other aspects of "lower cognition" that do indeed inform executive cognition, such as object classification.

Additionally, it is difficult to separate "knowledge" from the reasoning process that created it. A person's knowledge is not simply a compendium of declarative facts; it is also the relational or inferential semantic meaning a person gives the facts, patterns of facts and their relationships, the perceived worth of those facts, and the ways in which facts are used to reason about the encounter with future novel information. Indeed, facts are useless without the reasoning power to manipulate them, and so we believe that the ' $\mathrm{K}$ ' submodel, must include the cognition processes that created it. 
Knowledge determines the methods used when new knowledge is integrated with the old. Van Wijk also seems to imply this in how he models his "user;" his model pictures Knowledge feeding Exploration. But $\mathrm{K}$ cannot inform $\mathrm{E}$ without the guiding focus of a reasoning process. Indeed, exploration itself is cognition in action.

With these thoughts in mind, it would be more representative if Perception, Knowledge, and Exploration were all modeled as cognitive processes informing each other. We would see $\mathrm{P}$ as the early cognitive processes of selective attention, categorization, accommodation, including perceptual logic. (See Section 2.1.) $\mathrm{K}$ is viewed as meaningful knowledge with the use of reasoning, problem-solving and other thought processes that allow the human to create knowledge, and $\mathrm{E}$ as a focused, interactive cognitive process utilizing both $\mathrm{P}$ and $\mathrm{K}$.

When viewing the model this way, it's easy to see that two additional directional arrows need to be added to the model: from $\mathrm{P}$ to $\mathrm{E}$, and from $\mathrm{E}$ to $\mathrm{K}$. The first arrow indicates the important role that perception and perceptual logic plays in active exploration. The second arrow signifies how a rhythm of interaction feeds knowledge reasoning. As the human explores and learns, that learning directs and focuses the attention of further exploration. Additionally, van Wijk expressed Knowledge in this way:

$$
K(t)=K_{0}+\int_{0}^{t} \mathrm{P}(\mathrm{I}, \mathrm{K}, \mathrm{t}) \mathrm{dt} \quad[22]
$$

While this expression does encapsulate the idea that Perception is a vital part of the process, our integration would express the creation of knowledge over time more like the following:

$$
K(t)=K_{0}+\int_{0}^{t} \mathrm{E}(\mathrm{P}, \mathrm{K}, \mathrm{t}) \mathrm{dt}
$$

where Knowledge is the extension of currently held knowledge through the integrated perceptual and reasoning cognitive processes of Exploration. 


\section{Considering the Pirolli \& Card Sensemaking Model}

The HCM and its design implications share some understanding of the tasks involved in information processing with what is commonly called the "sensemaking model" as specified by Pirolli and Card [23]. This model identifies a series of tasks that are used progressively by analysts to find and filter evidence in the creation of hypotheses and drawing of conclusions.

The task series is presented as iterative, using both top down and bottom up cognitive tasks to forage and make sense of new information. The discussion of the foraging loop places emphasis on the human's ability to filter information and find patterns in the data. In the sensemaking loop, we see several tasks that are also the basis of the hypothesis generation subprocess of the HCM: listing hypotheses, listing evidence, and drawing conclusions.

The sensemaking model outlines processes involved in information processing. But to some degree, it treats these processes as a series of small black boxes, whose tasks are described but whose involved cognitive processes are largely undefined. One goal of the HCM is to identify and explore multi-layered cognition within information spaces and, in the process, to start opening up those black boxes so we can describe and find ways to support the processes they contain. Learning of whatever variety is not linear, but involves multiple subsystems that feed and inform each other throughout. Thus, it is important not to see human-visualization interaction as linear either, but rather as an interlinking of processes working in concert. 
Additionally, while the sensemaking model delineates cognitive processes between top-down and bottom-up processes, it does not handle cognition, which either does not fit cleanly into either group or which defies such categorization. One obvious example is what is sometimes called spontaneous insight, or insights that emerge in "a-ha" moments with seemingly little conscious preparation. What triggers and informs this variety of problem-solving is still the subject of debate $[24,25]$, but what is known suggests that spontaneous insight draws upon a wide scope of tacit knowledge, inferencing, and paradigm-discarding information reorganization. This reorganization leads to a profound understanding of the problem's solution, without the plodding trial-and-error or other incremental heuristics traditionally associated with problemsolving. Moreover, this understanding happens in what is often described as a "flash of time[25]," and, because much of the preparation is unconscious, the problem solver rarely can define where or how the solution came into focus.

More generally and in less dramatic fashion, human reasoning itself often defies the sensemaking model's incremental, bi-process structure. Reasoning is much more than a series of delineated tasks; depending on complexity, reasoning can combine any number of heuristics and use top-down and bottom-up thought constructions combinatorially. (For more discussion, please see [1].) Any generally-applicable model of human cognition must deal with this complexity sooner or later.

Briefly, we will discuss specific HCM theory and submodels that attempt to articulate the cognitive processes involved in the black box task processes of the sensemaking model. The foraging loop, which starts with a general, base categorization and moves downward into the more specific (the exploring-enriching-exploiting tradeoff) utilizes a variety of cognitive processes previously discussed, including the use of elimination heuristics and the use of 
mental models which lead to generalized inferencing rules. The foraging loop also implies (but without specifics) the behavior of direct interaction, which promotes rapid and responsive interaction, insulating the reasoning flow. On the other hand, the "leverage points" of the sensemaking loop described by Pirolli and Card [23] address hypothesis generation, which is also directly addressed by the HCM. In addition, the HCM uses mixed-initiative computer-aided discovery to address the biases that can confound evidentiary support needed in hypotheses analysis.

In conclusion, this discussion is not intended to propose the $\mathrm{HCM}$ as a replacement for the sensemaking model, but rather to join it with that operational model; the HCM is a step forward in the evolution of an understanding of holistic human cognition. Thus, our objective is to not only to outline the cognition involved in these processes, but to elucidate how that understanding can benefit and inform visualization design.

\section{EXAMPLES}

In this section, we will demonstrate the model guidelines by using them to evaluate and/or illustrate several visual analytics designs. The model, which was not used as a basis for these designs, provides a deeper understanding of the choices made and how the designs might be improved. Because we can discuss the rationale behind them more fully, we present predominantly designs that we helped develop. However, the arguments we make here would also apply to many other designs. 


\section{WireVis}

Discovering financial fraud in the great stream of transactions at a large bank is a difficult, timeconsuming, and expensive process since it must employ expert analysts and uncover everchanging modes of operation by criminals. The WireVis system was designed to combine the art of analysis with the science of visual analytics [26]. WireVis is an expert system, enhancing insight with what, in the terms of our Knowledge expression in the last section, is presumed to be the human's already sizeable K0; it provides intuitive visual representations of wire transactions, enhancing P; different views within the system allow the analysts to gain an overview of all transactions in one glance, while the ability to drill down into specific details of individual transactions enables finer examination and scrutiny. A time-based visualization allows the analysts to detect abnormal temporal patterns. Search by example permits selection of a particular keyword or time distribution pattern; the system then finds patterns that are similar to (or quite different from) the selected pattern. Finally, a keyword network shows keyword links for the selected group of transactions (where linked keywords appear in the same transaction), uncovering relationships that significantly aid the analyst in picking out suspicious transactions. This process highlights the importance of $E$ in extending K. Results of a user evaluation found WireVis to be an efficient, effective tool, which can discover suspicious patterns in a great mass of transaction data [26]; the tool is also generally applicable to other types of transactional analysis 
WireVis has a number of capabilities that conform to the above cognitive model and highlights some of the design choices that must be made. Four windows are tailored to specific, important views and tasks. Though having a single window to focus the user's attention may be ideal in some conceptual sense, and there is presumably a cognitive and perceptual load during task switching, multiple windows seem necessary for many complex analytical problems [27, 28]. The key is to minimize the load in order to mitigate the interference to the human's reasoning flow. This is done to a great extent through the concept of balanced interaction, as discussed next.

In WireVis, the views were carefully chosen so that overviews of main aspects of financial analysis were maintained. Linking and brushing between all views was enacted and immediate update to any interaction was enforced. (There are not only perceptual but cognitive aspects to maintaining high interactivity.) In addition, WireVis is designed to promote balanced interaction, during which the multiple interlinked windows act and look like a single interface, rather than separate entities; changes in one window are reflected across the interface, allowing the human to focus on the interaction; and similar types of interaction are supported in all windows. Thus, various selecting, filtering, and drill-down (through the transaction hierarchy of transactions) interactions appear simultaneously in the multiple windows. 
Further, very lightweight cursor passover interaction is enabled in several places (for example, passing over specific keywords). Finally, direct manipulation is used wherever possible to maintain user focus. We believe that balanced interaction is an essential design principle to keep investigators "in the cognitive zone" when using a multi-window interface on complex problems. With balanced interaction, the different components of the interface merge into one cognitive whole where, as one of the papers co-authors remarked, "The interaction is the analysis" [29]. WireVis also has search by example, which has been singled out in our cognitive model because it is very general and it keeps the user in the context of her reasoning process without interrupting it to construct the appropriate search query, which can quite difficult to accomplish. In this case, the user selects the keyword or transaction pattern she is thinking about to gather similar or dissimilar patterns. Search by example has been considered generally useful in other types of visualization, such as image analysis [30], broadcast news event analysis [31], and terrorism event investigation [32]. In fact, we believe that search by example should be part of any visual analytics interface involving analysis or reasoning tasks for large amounts of information.

\section{Jigsaw}

Jigsaw is a visual analytics system used to support investigative analysis [27]. It works with large collections of reports or other text documents and with the entities extracted from them. Its two main goals are to permit investigators to handle efficiently and move quickly through large document collections and to support hypothesis formation and evidence gathering. Jigsaw won the university portion of the VAST 2007 Contest, which centered on a simulated investigation similar to those carried out by intelligence analysts. 
As with WireVis, Jigsaw makes strong use of multiple windows with carefully tailored representations for complex investigative problems. The user is thought be in an "information cockpit" with multiple monitors, in front of and above the user [27]. Jigsaw seeks to maximize pixel use to take advantage of both the user's high acuity central focus and wide peripheral field. This is also a valid design point for WireVis (which requires at least two desktop monitors or a high resolution cinema display) or any other multi-window system.

However, although Jigsaw has some linking and brushing to integrate the windows, it does not have the balanced interaction WireVis employs. Based on the HCM guidelines, we would expect that users of Jigsaw would be less in the flow and require more cognitive effort than in WireVis during window management and connection. This is certainly a point worthy of further analysis and evaluation.

As a point of contrast, Jigsaw uses a bottom-up approach, employing an incremental, querybased method to bring in subsets of data for exploration and possible connection, as compared with WireVis's top-down visualization of the whole dataset and its context. Undoubtedly both approaches are valid and could be available in a general tool for complex problem-solving, and will be the subject of future study. Finally, Jigsaw is usable and simple. The interface permits direction interaction with representations of entities and reports, changing focus and detail. As with WireVis and other tools described here, simplicity and intuitiveness are not just goals based on perception principles but also based on the need for cognitive support. The HCM provides a point of view for investigating these goals in that light. 


\section{UrbanVis}

UrbanVis is a system created to combine views of detailed geographical information with relational views of associated abstract information [33]. The geographical view is based on urban legibility theories in architecture, and the overall system permits the user to interactively explore an urban environment to understand the detailed characteristics of a city.

As with WireVis and Jigsaw and for the same general reasons, UrbanVis is highly dependent upon multiple views, with a 3D multiresolution map driving the updates of a straight category view and a parallel coordinates view, giving the user a rich overview of many categories and relations at once. These views were carefully chosen after consultation with architects, urban planners, and GIS experts. UrbanVis provides a general approach to reasoning with the combination of geographic and abstract data. We are applying UrbanVis to bridge management data over city and state regions and are planning to use it for situation awareness in emergency response. This and the other examples in Section 5 show the generality of a multi-window approach that is designed with principles of human cognition in mind. Users of UrbanVis interact directly with the information, moving a geographic pointer and highlighting areas of interest or conversely choosing categories or individual coordinates in the parallel coordinates view to highlight specific geographic areas. This makes it easer to discover hidden geographical patterns for combinations of demographic or other abstract data. In the same sense as with WireVis, UrbanVis provides balanced interaction. This combined with direct manipulation, makes interaction the central focus. In addition, UrbanVis also provides a top-down, exploratory view with drill down controlled by simple movement of the ball up and down. The interface has only one menu, as it strives to keep the user cognitively focused during problem solving. 
Finally, since UrbanVis utilizes the central role of interaction, the visualization makes $\mathrm{E}$, as defined in Section 4, the seminal focus. In this interface, designed for a broad cross-section of users, $\mathrm{KO}$ can be small or great, and an attempt is made through use of color and spatial organization to facilitate $P$.

\section{GVis}

Although the human is uniquely qualified for "higher order" reasoning, our human cognition model permits the computer to support this process in numerous ways. GVis in this section and SRS in the next provide some of this support. As several of the visualization approaches utilize similar methods, in these final sections we will highlight areas that are different from WireVis. Using information available in public biological databases such as NCBI, GVis pictures the relationships and publications known about genomic data [34]. Due to the detail inherent in genomic data, the amount of information presently viewable during drill down in direct interaction becomes quickly overwhelming; the use of multiple views to visualize multiple levels of information hierarchies prevents humans from "losing their place" in the taxonomy. Also, similarly to WireVis, GVis is an expert visualization, requiring a rather sizeable $\mathrm{KO}$ to focus effective Exploration. This may mitigate the cognitive overload effects of information on $\mathrm{P}$ and $\mathrm{K}$ in Figure 2. A popup menu allows the user to view and explore publications on the spheres in the main view. This is perhaps not an optimum solution, as use of the menu is not intimate and can obstruct the field of view, which could threaten reasoning flow insularity and reduce the value of direct interaction. 
The visualization uses color and simple circles to highlight groups, insulating reasoning flow and focusing P. In addition, it employs the notion of a stretchable canvas, similar to Pad++ [30], to handle detail at all scales. The latest version of GVis applies the precepts of knowledge visualization, relying on taxonomic and ontological representations of genomic knowledge to determine what to visualize for the task at hand. When combined with the stretchable canvas, important knowledge at any scale can be made visible to support the current reasoning task. Thus, for example, glyphs showing how many genes are annotated (and what types) are made visible at the genome or even the cluster level, even though the individual genes are sub-pixel at these levels. This provides important support for the human reasoning process.

\section{Scalable Reasoning System (SRS)}

In SRS, Pike et al. demonstrate nicely the capacity of visual analytics to aid in hypothesis generation and analysis [35]. For example, while searching by example in SRS is limited to text searches, queried information can become "reasoning artifacts" to be used as the basis of hypotheses, or as evidence for hypotheses represented in "thought chains." The human is free to manipulate these artifacts directly in a sandbox-like information space, which encourages reasoning flow insularity and focuses $P$ as described in Section 4. Additionally, by allowing the human to arrange artifacts, interaction becomes the principle objective. While SRS does not use multiple views to display information, by allowing rearrangement of artifacts, SRS encourages the human to organize them in a way that is meaningful to the individual. Additionally, SRS is more than a display. New knowledge can be created by creating relationships between reasoning artifacts. Thus, as the human generates hypotheses and their evidence, new knowledge that is created during the process is not lost, either to the current analysis, or to all other humans given editable confidence ratings, which aids in the weighing of evidence in hypothesis analysis. 


\section{TOWARDS A PREDICTIVE HUMAN COGNITIVE MODEL}

The current HCM is of substantial use in providing a framework for understanding the humancomputer interface, in providing some initial design principles for both visual representation and interaction that can then be evaluated, and in revealing what to evaluate to support human cognition. However, the HCM can be made many times more powerful by making it predictive. The GOMS model, for example, is a predictive model, based on $\mathrm{HCl}$ evaluation research, and is derived for the design of mouse-driven, window interfaces [38]. It demonstrably improved the speed of the design process for these systems because it permitted the designer to avoid the slow, laborious process of user studies for each element of the design and for the overall layout. Indeed, because of its predictive capability, it permitted optimization of these interfaces beyond what could be accomplished with painstaking user studies because the design space could be explored in a comprehensive, predictive manner.

To develop a predictive HCM, we start with the idea of the user being in, falling out of, and regaining the cognitive zone (hereafter, referred to as the CZ). Initially, we want to develop a phenomenological model of these effects. We concentrate on two predictive mechanisms within the $\mathrm{HCM}$, both focused on keeping the user in the $\mathrm{CZ}$ and in a high state of reasoning efficiency. The first predicts the cost and benefit of design principles and decisions for keeping the user within the HCM, including the cost of having to regain this state after falling out of it. The second predicts the enhanced (or depressed) probability of making a discovery and gathering an insight in a given period of time. 
This approach reflects two aspects of the human reasoning process. The first part is performance-oriented; the more effort spent on a reasoning task (measured by number of operations or some other measure), the more will be accomplished. The second part attempts to capture spontaneous insight. It is not as dependent on number of operations and may even be diminished by requiring too much focus and structure from the participant [39].

We construct a cost/benefit model for the first part of our approach. Cost/benefit models have wide applicability and, in particular, have been used successfully on adaptive graphics rendering [40] and in van Wijk's work, discussed previously [22]. A cost/benefit model would be (following van Wijk's approach):

$G=n m W(\Delta K)^{\prime}$

$F=G-C=n m\left(W(\Delta K)-C_{S}-k C_{e}\right)-C_{i}-n C_{u}$

where $G$ is the benefit due to increased knowledge from use of the interactive visualization, $W$ is the value of the acquired knowledge, and $F$ is the profit. These quantities depend on the change in knowledge $\Delta K$, the number of users $n$, the number of sessions per user the data are visualized $m$, and the number of exploratory steps per visualization $k$. Costs depend on the initial development cost $C_{i}$, the initial cost per user $C_{u}$ (e.g., for selecting, tailoring, and learning how to use the visualization), the initial cost per session $C_{s}$ (e.g., for converting data and setting initial specifications), and the perception/exploration/cognition cost $C_{e}$ (e.g., the user must spend time watching and understanding the visualization as the interactive exploration proceeds). 
Note that this model has a way to handle overall knowledge gain by a number of users (say, in a collaborative setting or for a tool that is widely used by a number of people) and takes into account training effort and costs per session for things such as data setup. Of course, the model is limited. For example, it assumes that if a user repeatedly revisits a dataset for further exploration, his knowledge will continue to increase. But, even recognizing these limitations, a predictive model would still be a powerful tool. Here, we are most interested in assessing the terms $G$ and $C_{e}$. For the latter, we assess exploration costs and the penalty for falling out of the $\mathrm{CZ}$ and having to regain one's cognitive rhythm for the task at hand. Here we will need to categorize and rank actions. It is undoubtedly true that some actions are more disruptive than others, and the HCM provides us guidance as to what those are. Then we can plan evaluation strategy for quantifying, at least roughly, both $G$ and $C_{e}$. We have begun some of those evaluations, which will be reported elsewhere.

To capture discovery and spontaneous insight, we will need a separate probabilistic model. Our first steps toward this model are necessarily crude; there is little in the literature on modeling these processes. The main aspect we wish to capture is the disruptive effect to flashes of insight due to falling out of the $\mathrm{CZ}[39,40]$, as well as the ameliorative effect of staying within the zone. Our basic assumption within this model is that, using the $\mathrm{HCM}$, we can distinguish operations that keep the user in the $\mathrm{CZ}$ from those that are disruptive. To represent the cumulative effect of non-disruptive operations that lead to insight formation within the CZ (e.g., exploratory operations that support reasoning), we assume a probabilistic function that grows and then levels off over time as the user employs the interface. (See Figure 3 below.) However, there can be disruptive actions that take the user out of the CZ (e.g., having to form a Boolean search query 
or use a pull-down menu). These are represented by discontinuous downward trends, represented by breaks in the probability curve (as in Figures $3 b$ and $3 c$ ), whose depth is determined by the degree of disruption. The idea here is that the probability of gaining a spontaneous insight over a given period of time can be significantly lowered by significant disruptions or repeated disruptions. We can determine a rough shape for this probabilistic function over time through controlled experiments that seed data with insights to be discovered (for example, several hidden, new patterns of fraud in wire transaction data within a data visualization) and then permit users to use a visual analytics tool to discover them. In this approach, the HCM provides a framework for categorizing and linking together the operations of the tool in terms of cognitive processes.
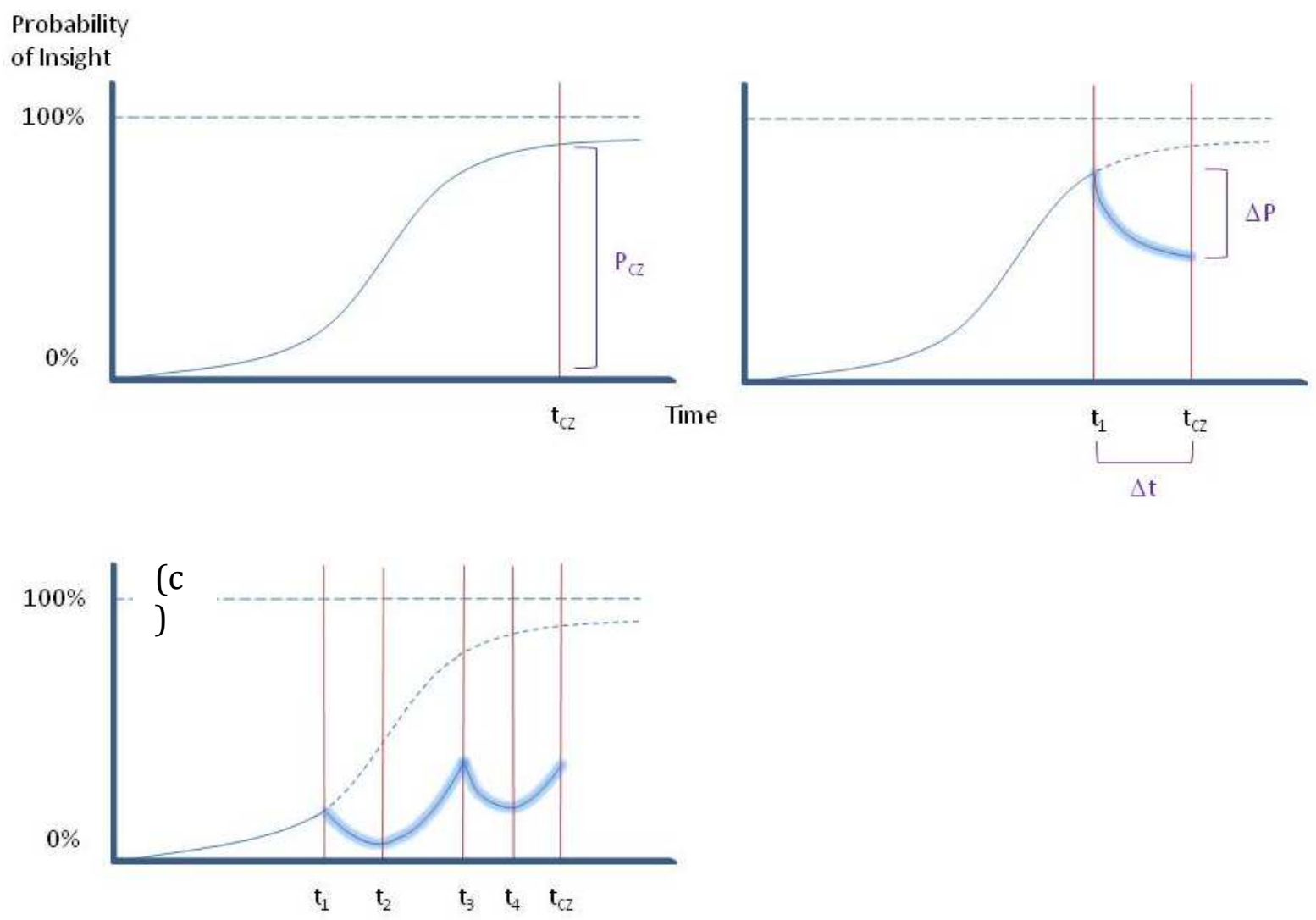

Figure 3. Probabilistic model for capturing discovery and spontaneous insight. 


\section{CONCLUSIONS}

If we in the visual analytics community are to attain our aspiration of more effective, more human-perceptive visualizations, we must begin to understand how humans manipulate semantically-complex information. It is not enough to determine what is being seen and given attention. Nor is it appropriate to infer combinatorial, individually-variable reasoning heuristics

from more binary cognitive behaviors. Just as an understanding of perceptual cognition based on evaluative research has been employed in creation of effective information visualization displays, a competent comprehension of reasoning, problem-solving, and decision-making must be employed in the development of mixed-initiative analytical interfaces.

What we have proposed is not a full working model, but is, as yet, a framework of human cognition. Our goal is to sketch out a system of "thinking about thinking" as a first step to interface interaction which is no longer just between user and data, but between human and computer partners, collaborating in the discovery of information and the creation and extension of knowledge.

What we have proposed is a model still in its rudimentary stages, whose future will undoubtedly be marked by additions, corrections, and multiple series of empirical evaluation. In some ways, this work still has the emergent expectation and general outline of an archaeological excavation; we cannot pretend to have all of the answers, but we know we're digging at the right spot. 
Even in its infant stages, the HCM has the potential to revolutionize how cognition is considered and evaluated in visualization design. For too long, visual analytic research has depended on black box assumptions about complex cognition that tend to be anecdotal, over-simplified, and non-generalizeable. And because of a broad lack of standardization in protocol and incomplete results reporting in published visual analytics evaluations, it is difficult or impossible to replicate results or build on prior work. Claims are made, but the discipline as a whole stands still. For these reasons, among others, the study of reasoning behaviors during interactive visualization is often difficult and unclear. This threatens to stymie progress; our ability to visualize is outstripping our knowledge of the thinking system we are visualizing for.

Additionally, the HCM reminds us that human cognition is not, nor shall it ever be, one-size-fitsall. One reason that reasoning cognition is complicated is that its combinatorial nature allows for maximal problem-fit and solution scalability. In addition, which heuristics are used often depends on innate and learned differences within reasoners, as well as the challenges of the presented task. The potentially exponential scale of this complexity seems daunting. But humans tend toward the habitual; they also prefer not to think harder than they must. Psychological sciences have used these tendencies to their advantage, grouping reasoners based on learning styles and other built-in preferences, and using these groupings to develop trait rubrics that aid in explaining and, in some cases, predicting future behavior. There is no reason that we could not build on this extant work and group users based on individual differences, domain similarities, and interaction behavior, provided we are willing to adopt a similar, exacting, experimental rigor. In fact we have begun studies in this direction where we compare the strategies of users of our tools based on whether they are expert or novice or based on their demonstrated problem-solving abilities. In the former case, we are finding that the higher level strategies are easier to pick out and analyze because our tools are designed and evaluated with the HCM in mind. 
One of the real strengths of creating a model such as the HCM is its inherent falsifiability. It would have been easier, perhaps, to choose one assumption, test and retest, then chose another assumption, test and retest, and perhaps eventually arrive at a model construct inductively. But the likelihood is that the trees would have muddled our sense of direction in the proverbial forest of mixed-initiative interaction. And very little would have been accomplished. By feeling our way to the boundaries of the forest, and marking its perimeter, and through application of insight, we have a better sense of where we are. The HCM is itself a hypothesis based on the evidence of cognitive research and insights derived from the visual analytics designs of ourselves and others. But when it is applied, it informs the questions we ask and the evaluations we design. It provides a clarifying framework and a way to organize the results we get when we use and analyze our visual analytics tools. And it reminds us that individual cognitive behaviors must be evaluated within a holistic context. It is a place to start, and it is a thing to be tested. And as with all hypotheses, our testing will show what is valid and what needs to be expanded upon or changed.

We have been able to show extant examples of several of the HCM's submodels, but there is still work to be done. There are, as yet, no spontaneous methods of searching by analogical structure. Computer-aided discovery will require both a better understanding of learning interfaces as well as a comprehensive understanding of human iterative thought chaining. There is also the pesky problem of a unified theory of reasoning. Understanding the available fragmented research is a good foundation, but we must approach a more complete discernment of how all of the pieces work together in an information space to be better able to define and evaluate the best ways to insulate reasoning flow, mitigate cognitive load, facilitate appropriate task switching, and minimize attentional interference during the reasoning process. 
Finally, there is the issue of better understanding of the temporal coordination of human reasoning and computation and presentation of information. The temporal dynamics of control actions and cognitive processing were addressed early in the history of $\mathrm{HCl}$ with $\mathrm{GOMS}$ and keystroke analysis. However the dynamic coupling of scene gist perception, eye movements, attentional allocation, cognitive processing and microstrategic perception/action patterns [36]

remains to be explored. Recent advances in the application of nonlinear dynamical modeling[37] may provide sufficient predictive validity for incorporation into models of sensemaking in visual analytics.

These tasks are broad items on a bold agenda. But our evaluation has also uncovered multiple practical problems, and directed the search for how best to tackle them: what number of multiple views maximizes the cognitive return on investment, the best way for the computer to suggest unconsidered information without interrupting - or annoying - the human at work, and methods of maintaining the interactive process so as to keep cognition in the flow, whatever the task. Finally, it is also clear that there must be strong support for permitting and managing human annotations. But again, it all starts with daring to peek into reasoning's black box.

\section{ACKNOWLEDGEMENTS}

This work was performed with support from the National Visualization and Analytics Center (NVACTM), a U.S. Department of Homeland Security Program, under the auspices of the SouthEast Regional Visualization and Analytics Center. Special thanks to Benjamin F. Green for helpful insights. 


\section{REFERENCES}

[1] Green TM, and Ribarsky W. Using a human cognition model in the creation of collaborative knowledge visualizations. SPIE Defense + Security 2008 (Orlando, FL).

[2] Newell A. You can't play 20 questions with nature and win: Projective comments on the papers of this symposium. In Visual Information Processing. Chase, W.G. (Ed). New York: Academic Press, 1973.

[3] Piaget P. "Piaget's theory." In Cognitive development to adolescence, K. Richardson, and S. Sheldon, (Eds.) Erlbaum: Hillsdale, NJ, 1988; 3-18.

[4] Komatsu KL. Recent views of conceptual structure. Psychological Bulletin 1992; 112: 500 526.

[5] Gigerenzer G and Goldstein DG, Reasoning the fast and frugal way: Models of bounded rationality. Psychological Review 1996; 103(4).

[6] Biederman I. Recognition by components: A theory of human image understanding. Psychological Review 1987; 94 (2): 115 -147.

[7] Rensink RA. A dynamic representation of scenes. Visual Cognition 2000; 7.

[8] Pylyshyn Z, Seeing and Visualizing: It's not what you think, MIT Press/Bradford Books, Cambridge, MA. 2003.

[9] Pylyshyn Z. Things and Places. IT Press/Bradford Books, Cambridge, MA. 2007.

[10] Ullman S. Visual routines. Cognition 1984. 97-159.

[11] Rock I. The logic of perception. MIT Press/Bradford Books, Cambridge, MA. 1985.

[12] Tarr MJ. Learning to see faces and objects. Trends in Cognitive Sciences 2003. 7(1).

[13] Kozielecki J. Elements of a psychological decision theory. Studia Psychologica 1971. 13(1): 53-60.

[14] Cherubini P and Mazzocco A. From models to rules: Mechanization of reasoning as a way to cope with cognitive overloading in combinatorial problems. Acta Psychologica 2004. 16(3): 223-243. (2004).

[15] Miller GA. The magic number seven, plus or minus two: Some limits on our capacity for processing information. Psychological Review (63). 81-97.

[16] Wason PC. On the failure to eliminate hypotheses in a conceptual task. Quarterly Journal of Experimental Psychology 1960. 12: 129-140.

[17] Evans J.St. BT, Varston J, and Pollard P, On the conflict between logic and belief in syllogistic reasoning. Memory and Cognition 1983. 11: 295-306. 
[18] Baldonado MQW, Woodruff A, and Kuchinsky A. Guidelines for using multiple views in information visualization. In Proceedings of Working Conference on Advanced Visual Interfaces 2000 (Palermo, Italy).

[19] Bederson BB, Hollan JD. Pad++: A zooming graphical interface for exploring alternate interface physics. In UIST 1994: 17-26.

[20] Pirolli P. Computational models of information scent- following in a very large browsable text collection. Proceedings of the Conference on Human Factors in Computing Systems, $\mathrm{CHI}$ 1997 (Atlanta, GA).

[21] Heuer RJ, The Psychology of Intelligence Analysis. Center for the Study of Intelligence. CIA. 1999.

[22] van Wijk JJ, The value of visualization. IEEE Visualization, Proceedings of Vis 2005: 79 86.

[23] Pirolli P and Card S. The Sensemaking Process and Leverage Points for Analyst Technology as Identified Through Cognitive Task Analysis. Proceedings of International Conference on Intelligence Analysis 2005: 2-4.

[24] Knoblich G, Ohlsson S, and Raney GE. An eye movement study of insight problem solving. Memory and Cognition 2001 29(7): 1000-1009.

[25] Bowden EM, Jung-Beeman M, Fleck J, and Kounios J. New approaches to demystifying insight. Trends in Cognitive Sciences 2005 9(7):322-328.

[26] Chang R, Ghoniem M, Kosara R, Ribarsky W, Yang J, Suma E, Ziemkiewicz C, Kern D, Sudjianto A. WireVis: Visualization of categorical, time-varying data from financial transactions. Proceedings of the 2007 IEEE Symposium on Visual Analytics Science and Technology 2007 (Sacramento, CA): 155-162. IEEE Computer Society.

[27] Stasko J, Gorg C, Liu Z and Singhal K, Jigsaw: Supporting Investigative Analysis through Interactive Visualization. Proceedings of 2007 IEEE Symposium on Visual Analytics Science and Technology 2007 (Sacramento, CA): 131-138.

[28] Wise JA, Thomas JJ, Pennock K, Lantrip D, Pottier M, Schur A, and Crow V. Visualizing the non-visual: spatial analysis and interaction with information from text documents. In INFOVIS 1995: Proceedings of the 1995 IEEE Symposium on Information V visualization: 51. IEEE Computer Society.

[29] Private communication, Remco Chang.

[30] Yang J, Fan J, Hubball D, Gao Y, Luo H, Ribarsky W, and Ward M. Semantic Image Browser: Bridging Information Visualization with Automated Intelligent Image Analysis. Proceedings of IEEE VAST 2006: 191-198.

[31] Ghoniem M, Luo D, Yang J, and Ribarsky W. NewsLab:Exploratory Broadcast News Video Analysis. IEEE VAST 2007: 123-130. 
[32] Wang X, Chang R, Kosara R, Ribarsky W, Smarick K, and Miller E. Investigative Visual Analysis of Global Terrorism. EG/IEEE EuroVis 2008.

[33] Chang R, Wessel G, Kosara R, Sauda E, and Ribarsky W. Legible Cities: FocusDependent Multi-Resolution Visualization of Urban Relationships. IEEE Transactions on Visualization and Computer Graphics (TVCG) InfoVis 2007 (Sacramento, CA).

[34] Hong J, Jeong DH, Shaw CD, Ribarsky W, Borodovsky M, and Song C. GVis: A Scalable Visualization Framework for Genomic Data," In Proceedings of EuroVis 2005: 191-198.

[35] Pike AP, May R, Baddeley B, Riensche R, Bruce J, and Younkin K, Scalable visual reasoning: supporting collaboration through distributed analysis. Proceedings of IEEE International Symposium on Collaborative Technologies and Systems 2007.

[36] Gray WD, and Boehm-Davis DA. Milliseconds Matter: An introduction to microstrategies and to their use in describing and predicting interactive behavior. Journal of Experiment Psychology: Applied 2000 6(4): 322-335.

[37] Spivey M. The Continuity of Mind. Oxford Press, NewYork, NY; 2007.

[38] Card SK, Moran TP, and Newell A. The Psychology of Human-Computer Interaction. Lawrence Erlbaum, Hillsdale, N.J: 1983.

[39] Lehrer J. The Eureka Hunt: Why do good ideas come to us when they do? New Yorker, July 28, 2008: 40.

[40] Wilson TD, and Schooler JW. Thinking too much: introspection can reduce the quality of preferences and decisions. Journal of Personality and Social Psychology 1991 60(2): 181-192.

[41] Tera Green, William Ribarsky, and Brian Fisher. Visual Analytics for Complex Concepts Using a Human Cognition Model. Proc. IEEE VAST 2008, pp. 91-98. 


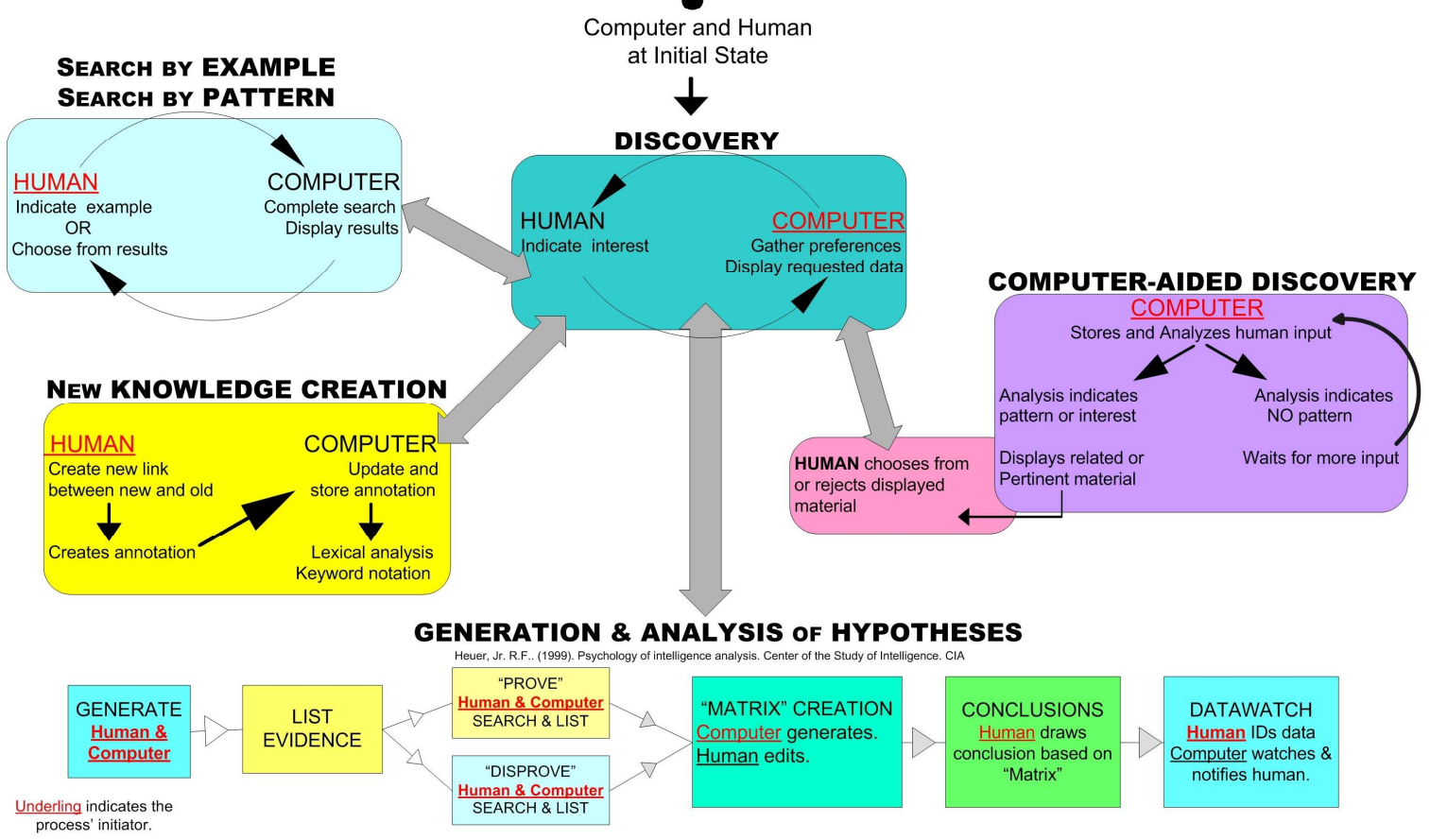

Figure 1. The Human Cognition Model (HCM) showing process initiators.

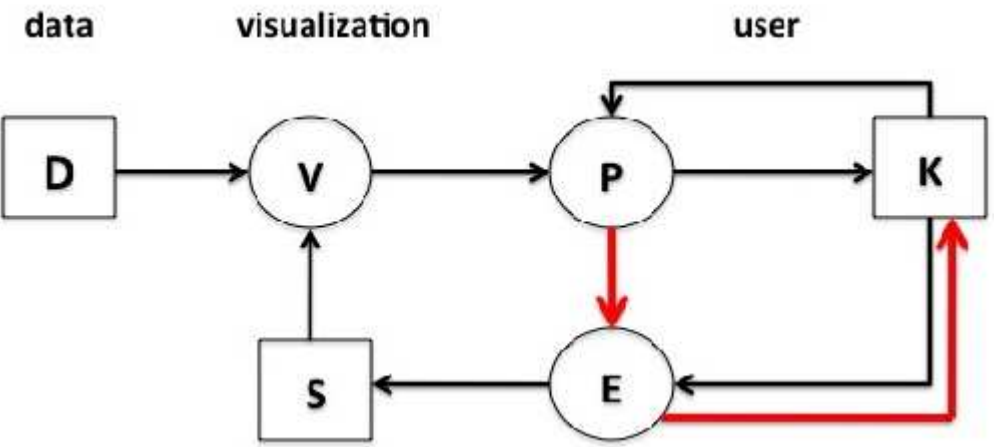

Figure 2. The van Wijk visualization model, with our integrations in red. 